\title{
LORENTZ SPACES THAT ARE ISOMORPHIC TO SUBSPACES OF $L^{1}$
}

\author{
CARSTEN SCHÜTT
}

ABstract. We show which Lorentz spaces are isomorphic to subspaces of $L^{1}$ and which are not.

The class of subspaces of $L^{1}$ is known to be quite big. Even if one considers only symmetric subspaces of $L^{1}$, i.e. subspaces that have symmetric basis or that are isomorphic to a rearrangement invariant function space, one finds that the class is quite rich.

It can be shown that those spaces are isomorphic to averages of Orlicz spaces [3, 11]. It was first believed that those spaces are in fact just Orlicz spaces [1]. Examples were given that this is not the case [3]. These examples have an abstract nature. Therefore our paper serves two purposes. On one hand it decides the natural question, which Lorentz spaces embed into $L^{1}$ and on the other hand it gives some examples of spaces that show up naturally in functional analysis and that are symmetric subspaces of $L^{1}$ though they are not Orlicz spaces.

In particular we show that the Lorentz spaces $L^{p, q}$ embed into $L^{1}$ if and only if $1 \leq q \leq p<2$ or $p=q=2$.

We would like to mention that infinite-dimensional versions of the Orlicz spaces that we use in our standard embedding were already used in [6] for some other purpose. This type of space was first studied by Rosenthal [12].

In $\S 1$ we state the theorem and derive some corollaries. Also we present some propositions from which the theorem follows. The propositions are proved in the following section.

\section{Preliminaries}

A basis $\left\{e_{i}\right\}_{i=1}^{n}$ of a normed space is $C$-symmetric if we have for all signs $\varepsilon_{i}= \pm 1, x_{i} \in \mathbf{R}$, and all permutations $\pi$

$$
\left\|\sum_{i=1}^{n} x_{i} e_{i}\right\| \leq C\left\|\sum_{i=1}^{n} \varepsilon_{i} x_{\pi(i)} e_{i}\right\| .
$$

Received by the editors June 1, 1987. Presented at the AMS meeting, Kent, Ohio, April 3-4, 1987.

1980 Mathematics Subject Classification (1985 Revision). Primary 46B20, 46B25.

Research supported by NSF Grant DMS 86-02395. 
The Banach-Mazur distance of two Banach spaces $E$ and $F$ is $d(E, F)=$ $\inf \left\{\|I\|\left\|I^{-1}\right\| I\right.$ is an isomorphism between $E$ and $\left.F\right\}$. For a given $x \in \mathbf{R}^{n}$ the sequence $x_{i}^{*}, i=1, \ldots, n$, is the decreasing rearrangement of $\left|x_{i}\right|, i=$ $1, \ldots, n$. If $f$ is a measurable function on $[0,1]$ we denote by $f^{*}$ the decreasing rearrangement of $|f|$. Let $1=a_{1} \geq a_{2} \geq \cdots \geq a_{n} \geq 0$. The Lorentz space $d(a, p)$ is $\mathbf{R}^{n}$ with norm

$$
\|x\|=\left(\sum_{i=1}^{n} a_{i}\left|x_{i}^{*}\right|^{p}\right)^{1 / p}
$$

and, if $W$ is a nonincreasing, continuous, nonnegative function on $(0,1]$ with $\int_{0}^{1} W(t) d t=1$, the Lorentz space $L_{W}^{p}[0,1]$ is the space of measurable functions with norm

$$
\|f\|=\left(\int_{0}^{1}\left|f^{*}(t)\right|^{p} W(t) d t\right)^{1 / p} .
$$

A Banach space has cotype 2 with constant $C$ if we have for all sequences of vectors $x_{i}, i=1, \ldots, n$,

$$
\left(\sum_{i=1}^{n}\left\|x_{i}\right\|^{2}\right)^{1 / 2} \leq C \mathrm{Ave}_{ \pm}\left\|\sum_{i=1}^{n} \pm x_{i}\right\|
$$

and a Banach lattice is 2-concave with constant $C$ if

$$
\left(\sum_{i=1}^{n}\left\|x_{i}\right\|^{2}\right)^{1 / 2} \leq C\left\|\left(\sum_{i=1}^{n}\left|x_{i}\right|^{2}\right)^{1 / 2}\right\| .
$$

For a given Banach space we denote the infimum of these numbers $C$ by concave $_{2}(E)$.

\section{THE THEOREM}

Theorem 1.1. Let $1 \leq p<2$ and $W$ be a nonincreasing, positive, continuous function on $(0,1]$ with $\int_{0}^{1} W(t) d t=1$. Then the following are equivalent:

(i) $L_{W}^{p}$ is isomorphic to a subspace of $L^{p}$.

(ii) $L_{W}^{p}$ has cotype 2 (is 2-concave).

(iii) There is a $C>0$ such that we have for all $x \in[0,1]$

$$
\int_{0}^{x} W(t) t^{-p / 2} d t \leq C x^{-p / 2} \int_{0}^{x} W(t) d t .
$$

Proposition 1.2. Let $1 \leq p \leq 2$ and $1=a_{1} \geq a_{2} \geq \cdots \geq a_{n} \geq 0$ such that

$$
a_{1}+\left(1-\frac{p}{2}\right) \sum_{j=1}^{k} a_{j} j^{-p / 2} \leq C k^{1-p / 2} a_{k}, \quad k=1, \ldots, n .
$$

Then there is a subspace $E$ of $l^{p}$ such that $d(E, d(a, p)) \leq C D$ where $D$ is a universal constant.

Proposition 1.2 will be proved in $\S 3$. 
Proposition 1.3. Let $1 \leq p<2$ and $1=a_{1} \geq a_{2} \geq \cdots \geq a_{n} \geq 0$. Then we have

$$
\sum_{i=1}^{k} a_{i} i^{-p / 2} \leq D_{p} k^{-p / 2} \sum_{i=1}^{k} a_{i}, \quad k=1, \ldots, n,
$$

where $D_{p}$ depends on $p$ and the 2-concavity constant of $d(a, p)$ but not on $n$.

Proposition 1.3 follows from Lemma 4.3 and Proposition 4.4 with $s=$ $2 /(2-p)$.

Lemma 1.4 $[9$, p. 306]. Let $X$ be a separable Banach space and $1 \leq p<\infty$ such that for every finite-dimensional subspace $E$ of $X$ there is a subspace $\widetilde{E}$ of $l^{p}$ with $d(E, \widetilde{E}) \leq d$. Then there is a subspace $Y$ of $L^{p}[0,1]$ such that $d(X, Y) \leq d$.

Proof of Theorem 1.1. If $L_{W}^{p}$ is isomorphic to a subspace of $L^{p}$ then $L_{W}^{p}$ has cotype 2 , or, which is equivalent $[4,10]$, is 2-concave. We show first that $L_{W}^{p}$ is isomorphic to a subspace of $L^{p}$ if there is a constant $C$ such that (1.1) holds. By Lemma 1.4 it is enough to show that the subspaces of $L_{W}^{p}$ spanned by the vectors $\chi_{[(i-1) / n, i / n]}, i=1, \ldots, n$, are uniformly isomorphic to subspaces of $L^{p}$. We have

$$
\left\|\sum_{i=1}^{n} x_{i} \chi_{[(i-1) / n, i / n]}\right\|_{L_{W}^{p}}=\left(\sum_{i=1}^{n}\left|x_{i}^{*}\right|^{p} \int_{(i-1) / n}^{i / n} W(t) d t\right)^{1 / p} .
$$

We put

$$
a_{i}=\int_{(i-1) / n}^{i / n} W(t) d t\left(\int_{0}^{1 / n} W(t) d t\right)^{-1}, \quad i=1, \ldots, n,
$$

and the space $d(a, p)$ is isometric to the subspace of $L_{W}^{p}$ spanned by

$$
\chi_{[(i-1) / n, i / n]}, \quad i=1, \ldots, n .
$$

By our hypothesis (1.1) we have

$$
\begin{aligned}
& \sum_{i=1}^{k} \int_{(i-1) / n}^{i / n} W(t)\left(\frac{i}{n}\right)^{-p / 2} d t \leq \int_{0}^{k / n} W(t) t^{-p / 2} d t \\
& \quad \leq C\left(\frac{k}{n}\right)^{-p / 2} \int_{0}^{k / n} W(t) d t=C\left(\frac{k}{n}\right)^{-p / 2} \sum_{i=1}^{k} \int_{(i-1) / n}^{i / n} W(t) d t .
\end{aligned}
$$

Therefore we get

$$
\sum_{i=1}^{k} a_{i} i^{-p / 2} \leq C k^{-p / 2} \sum_{i=1}^{k} a_{i}
$$

This means in particular

$$
\frac{1}{2} \sum_{i \leq k /(2 C)^{2 / p}} a_{i} i^{-p / 2} \leq \sum_{i \leq k /(2 C)^{2 / p}} a_{i}\left(i^{-p / 2}-C k^{-p / 2}\right) \leq C k^{-p / 2} \sum_{i \geq k /(2 C)^{2 / p}}^{k} a_{i} .
$$


Therefore we obtain

$$
\frac{1}{2} \sum_{i \leq k /(2 C)^{2 / p}} a_{i} i^{-p / 2} \leq C k^{1-p / 2} a_{\left[k /(2 C)^{2 / p}\right]} .
$$

This implies

$$
\sum_{i=1}^{k} a_{i} i^{-p / 2} \leq C^{\prime} k^{1-p / 2} a_{k}, \quad 1 \leq k \leq \frac{n}{(2 C)^{2 / p}},
$$

and $C^{\prime}$ depends only on $C$. In case this inequality does not hold for $k \geq$ $n(2 C)^{-2 / p}$ we simply change the $a_{k}$. We put

$$
\tilde{a}_{k}=a_{\left[n /(2 C)^{2 / p}\right]} \text { for } k>n(2 C)^{-2 / p} .
$$

By this we do not change the norm of $d(a, p)$ significantly. This follows by triangle-inequality. On the other hand we establish inequality (1.2) so that we can apply Proposition 1.2 to $d(a, p)$.

Now we show that (1.1) holds if $L_{W}^{p}$ has cotype 2 which is in particular true if $L_{W}^{p}$ is isomorphic to a subspace of $L^{p}$.

By $[4,10]$ a Banach lattice has cotype 2 if and only if it is 2-concave. If $L_{W}^{p}$ is 2-concave then the 2-concavity constants of the spaces $d(a, p)$ with $a$ defined by (1.3) have to be uniformly bounded. We apply Proposition 1.3 and get

$$
\sum_{i=1}^{k} a_{i} i^{-p / 2} \leq D_{p} k^{-p / 2} \sum_{i=1}^{k} a_{i}, \quad k=1, \ldots, n .
$$

Therefore we get

$$
\begin{aligned}
\int_{1 / n}^{k / n} W(t) t^{-p / 2} d t & =\sum_{i=2}^{k} \int_{(i-1) / n}^{i / n} W(t) t^{-p / 2} d t \\
& \leq \sum_{i=2}^{k}\left(\frac{i-1}{n}\right)^{-p / 2} \int_{(i-1) / n}^{i / n} W(t) d t
\end{aligned}
$$

By (1.3)

$$
=\int_{0}^{1 / n} W(t) d t \sum_{i=2}^{k}\left(\frac{i-1}{n}\right)^{-p / 2} a_{i} \leq \int_{0}^{1 / n} W(t) d t 2 \sum_{i=2}^{k}\left(\frac{i}{n}\right)^{-p / 2} a_{i} .
$$

By Proposition 1.3

$$
\leq \int_{0}^{1 / n} W(t) d t C_{p}\left(\frac{k}{n}\right)^{-p / 2} \sum_{i=1}^{k} a_{i}=C_{p}\left(\frac{k}{n}\right)^{-p / 2} \int_{0}^{k / n} W(t) d t .
$$

We obtain

$$
\int_{1 / n}^{k / n} W(t) t^{-p / 2} d t \leq C_{p}\left(\frac{k}{n}\right)^{-p / 2} \int_{0}^{k / n} W(t) d t
$$

for all $1 \leq k \leq n<\infty$. 
Corollary 1.5. The Lorentz spaces $L^{p, q}[0,1]$ are isomorphic to subspaces of $L^{1}[0,1]$ if and only if $1 \leq q \leq p<2$ or $p=q=2$.

For the proof we also require the following notion and lemma [8]. Suppose that $\left\{e_{i}\right\}_{i=1}^{n}$ is a 1 -symmetric basis of $E$. Then there is an Orlicz function $M_{E}$ such that

$$
\left\|\sum_{i=1}^{k} e_{i}\right\|_{E} \leq\left\|\sum_{i=1}^{k} e_{i}\right\|_{M_{E}} \leq 2\left\|\sum_{i=1}^{k} e_{i}\right\|_{E}, \quad k=1, \ldots, n .
$$

We say that $M_{E}$ is associated to $E$.

Lemma 1.6. Let $\left\{e_{i}\right\}_{i=1}^{n}$ be a 1-symmetric basis of $E$ and suppose that $E$ is $C$-isomorphic to a subspace of $L^{1}$. Then we have for all $x$

$$
\left\|\sum_{i=1}^{n} x_{i} e_{i}\right\|_{M_{E}} \leq C D\left\|\sum_{i=1}^{n} x_{i} e_{i}\right\|_{E}
$$

where $D$ is a universal constant and $M_{E}$ an associated Orlicz function.

Proof of Corollary 1.5. The positive direction for $1 \leq q \leq p<2$ is a consequence of Theorem 1.1. The negative direction follows from Lemma 1.6. The spaces $L^{2, q}$ do not have cotype 2 unless $q=2$ [2]. Therefore those spaces cannot be isomorphic to subspaces of $L^{1}$. If $p>2$ then $L^{p, q}$ does not have cotype $2[2]$.

\section{SOME REQUIRED LEMMAS}

Here we collect some results that were proved in other places and that are essential for the proof of the main result.

We prove the results by using averages over permutations. For other methods see $[6,12]$.

Lemma 2.1. Let $1 \leq p \leq 2$ and $a_{i j} \geq 0, i=1, \ldots, n, j=1, \ldots, N$, with $\sum_{i=1}^{n} \sum_{j=1}^{N}\left|a_{i j}\right|^{p}=n$. Then $\mathbf{R}^{n}$ with norm

$$
\|x\|=\left(\sum_{j=1}^{N} \operatorname{Ave}_{\pi}\left(\sum_{i=1}^{n}\left|x_{\pi(i)} a_{i j}\right|^{2}\right)^{p / 2}\right)^{1 / p}
$$

is $\sqrt{2}$-isomorphic to a 1-symmetric subspace of $l^{p}$.

Proof. Let $I$ be the isomorphism mapping $x \in \mathbf{R}^{n}$ onto an element from $l_{N 2^{n} n \text { ! }}^{p}$ given by $\left(2^{n} n !\right)^{-1 / p}\left(\sum_{i=1}^{n} x_{i} \varepsilon_{i} a_{\pi(i) j}\right)_{j, \ell, \pi}$. We have

$$
\|I(x)\|_{p}=\left(\sum_{j=1}^{N} \frac{1}{n !} \sum_{\pi} 2^{-n} \sum_{\varepsilon}\left|\sum_{i=1}^{n} x_{i} \varepsilon_{i} a_{\pi(i) j}\right|^{p}\right)^{1 / p} .
$$


By Khintchine-inequality we get

$$
\|I(x)\|_{p} \leq\left(\sum_{j=1}^{N} \operatorname{Ave}_{\pi}\left(\sum_{i=1}^{n}\left|x_{\pi(i)} a_{i j}\right|^{2}\right)^{p / 2}\right)^{1 / p} \leq \sqrt{2}\|I(x)\|_{p} .
$$

Lemma 2.2. Let $1 \leq p \leq q<\infty$, then we have

$$
\begin{gathered}
\frac{1}{10}\left\{\left(\frac{1}{n} \sum_{k=1}^{n}|S(k)|^{p}\right)^{1 / p}+\left(\frac{1}{n} \sum_{k=n+1}^{n^{2}}|S(k)|^{q}\right)^{1 / q}\right\} \\
\leq\left(\frac{1}{n !} \sum_{\pi}\left(\sum_{i=1}^{n}\left|a_{i \pi(i)}\right|^{q}\right)^{p / q}\right)^{1 / p} \\
\leq\left(\frac{1}{n} \sum_{k=1}^{n}|S(k)|^{p}\right)^{1 / p}+\left(\frac{1}{n} \sum_{k=n+1}^{n^{2}}|S(k)|^{q}\right)^{1 / q}
\end{gathered}
$$

where $S(k), k=1, \ldots, n^{2}$, is the decreasing rearrangement of $a_{i j}, i, j=$ $1, \ldots, n$.

Proof. The result for $p=1$ and a constant equal to $\frac{1}{5}$ is contained in [7]. To pass to the general case we consider the matrix $a_{i j}^{p}$ instead of $a_{i j}$. Also, as second exponent we have to choose $q / p$ which is possible since $p \leq q$.

As an immediate consequence we get the following lemma.

Lemma 2.3. Let $1 \leq p \leq 2$. Then we have for all $x \in \mathbf{R}^{n}$ and $k=1, \ldots, n$

$$
\begin{aligned}
C\left(\left(\sum_{i=1}^{k}\left|x_{i}^{*}\right|^{p}\right)^{1 / p}\right. & \left.+k^{1 / p-1 / 2}\left(\sum_{i=k+1}^{n}\left|x_{i}^{*}\right|^{2}\right)^{1 / 2}\right) \\
& \leq k^{1 / p}\left(\frac{1}{n !} \sum_{\pi}\left(\sum_{i \leq n / k}\left|x_{\pi(i)}\right|^{2}\right)^{p / 2}\right)^{1 / p} \\
& \leq\left(\sum_{i=1}^{k}\left|x_{i}^{*}\right|^{p}\right)^{1 / p}+k^{1 / p-1 / 2}\left(\sum_{i=k+1}^{n}\left|x_{i}^{*}\right|^{2}\right)^{1 / 2}
\end{aligned}
$$

where $x_{i}^{*}, i=1, \ldots, n$, denotes the decreasing rearrangement of $\left|x_{i}\right|, i=$ $1, \ldots, n$, and $C>0$ is a universal constant.

Proof. If we apply Lemma 2.2 to the matrix

$$
a_{i j}= \begin{cases}x_{i} & \text { if } 1 \leq j \leq n / k \\ 0 & \text { otherwise }\end{cases}
$$


we have

$$
\begin{gathered}
{\left[\frac{n}{k}\right] \sum_{i \leq k}\left|x_{i}^{*}\right|^{p} \leq \sum_{k=1}^{n}|S(k)|^{p} \leq \frac{n}{k} \sum_{i \leq k}\left|x_{i}^{*}\right|^{p},} \\
{\left[\frac{n}{k}\right] \sum_{i>2 k}\left|x_{i}^{*}\right|^{2} \leq \sum_{k=n+1}^{n^{2}}|S(k)|^{2} \leq \frac{n}{k} \sum_{i>k}\left|x_{i}^{*}\right|^{2} .}
\end{gathered}
$$

\section{THE STANDARD EMBEDDING}

Proof of Proposition 1.2. We consider $\mathbf{R}^{n}$ with norm

$$
\begin{array}{r}
\|x\|=\left\{\sum_{k=1}^{n-1}\left(a_{k}-a_{k+1}\right)\left|\left(\sum_{i=1}^{k}\left|x_{i}^{*}\right|^{p}\right)^{1 / p}+k^{1 / p-1 / 2}\left(\sum_{i=k+1}^{n}\left|x_{i}^{*}\right|^{2}\right)^{1 / 2}\right|^{p}\right. \\
\left.+a_{n} \sum_{i=1}^{n}\left|x_{i}\right|^{p}\right\}^{1 / p} .
\end{array}
$$

By Lemmas 2.1 and 2.3 these spaces are $C$-isomorphic to subspaces of $L^{p}$ where $C$ is a universal constant. We verify that this norm is equivalent to the norm of the $n$-dimensional Lorentz space $d(a, p)$. We have

$$
\begin{aligned}
\|x\| & \geq\left(\sum_{k=1}^{n-1}\left(a_{k}-a_{k+1}\right) \sum_{i=1}^{k}\left|x_{i}^{*}\right|^{p}+a_{n} \sum_{i=k}^{n}\left|x_{i}\right|^{p}\right)^{1 / p} \\
& =\left(\sum_{i=1}^{n}\left|x_{i}^{*}\right|^{p}\left(a_{n}+\sum_{k=i}^{n-1} a_{k}-a_{k+1}\right)\right)^{1 / p} \\
& =\left(\sum_{i=1}^{n}\left|x_{i}^{*}\right|^{p} a_{i}\right)^{1 / p} .
\end{aligned}
$$

On the other hand, by the same calculation we get

$$
\|x\| \leq\left(\sum_{i=1}^{n}\left|x_{i}^{*}\right|^{p} a_{i}\right)^{1 / p}+\left(\sum_{k=1}^{n-1}\left(a_{k}-a_{k+1}\right) k^{1-p / 2}\left(\sum_{i=k+1}^{n}\left|x_{i}^{*}\right|^{2}\right)^{p / 2}\right)^{1 / p}
$$

It is left to estimate the second summand. Since we have

$$
\left(\sum_{i=k+1}^{n}\left|x_{i}^{*}\right|^{2}\right)^{1 / 2} \leq\left(\left|x_{k}^{*}\right|^{p} \sum_{i=1}^{k} C_{i}+\sum_{i=k+1}^{n} C_{i}\left|x_{i}^{*}\right|^{p}\right)^{1 / p}
$$


with $\sum_{i=1}^{j} C_{i}=j^{p / 2}$ for $j=1, \ldots, n$ we get

$$
\begin{aligned}
& \left(\sum_{k=1}^{n-1}\left(a_{k}-a_{k+1}\right) k^{1-p / 2}\left(\sum_{i=k+1}^{n}\left|x_{i}^{*}\right|^{2}\right)^{p / 2}\right)^{1 / p} \\
& \quad \leq\left(\sum_{k=1}^{n-1}\left(a_{k}-a_{k+1}\right) k^{1-p / 2}\left(\left|x_{k}^{*}\right|^{p} \sum_{i=1}^{k} C_{i}+\sum_{i=k+1}^{n} C_{i}\left|x_{i}^{*}\right|^{p}\right)\right)^{1 / p} \\
& \quad \leq\left(\sum_{k=1}^{n-1}\left(a_{k}-a_{k+1}\right) k\left|x_{k}^{*}\right|^{p}\right)^{1 / p}+\left(\sum_{k=1}^{n-1}\left(a_{k}-a_{k+1}\right) k^{1-p / 2} \sum_{i=k+1}^{n} C_{i}\left|x_{i}^{*}\right|^{p}\right)^{1 / p}
\end{aligned}
$$

Since

$$
\sum_{k=1}^{j} k\left(a_{k}-a_{k+1}\right)=-j a_{j+1}+\sum_{k=1}^{j} a_{k} \leq \sum_{k=1}^{j} a_{k} \text { for } j=1, \ldots, n-1
$$

we can estimate the first summand by $\left(\sum_{k=1}^{n-1} a_{k}\left|x_{k}^{*}\right|^{p}\right)^{1 / p}$. Again, it is left to estimate the second summand

$$
\begin{aligned}
& \left(\sum_{k=1}^{n-1}\left(a_{k}-a_{k+1}\right) k^{1-p / 2} \sum_{i=k+1}^{n} C_{i}\left|x_{i}^{*}\right|^{p}\right)^{1 / p} \\
& \quad \leq\left(\sum_{i=1}^{n-1}\left|x_{i}^{*}\right|^{p} C_{i} \sum_{k=1}^{i}\left(a_{k}-a_{k+1}\right) k^{1-p / 2}+C_{n}\left|x_{n}^{*}\right|^{p} \sum_{k=1}^{n-1}\left(a_{k}-a_{k+1}\right) k^{1-p / 2}\right)^{1 / p} .
\end{aligned}
$$

We have

$$
C_{1}=1, \quad C_{k}=k^{p / 2}-(k-1)^{p / 2} \leq \frac{p}{2}(k-1)^{p / 2-1} \quad \text { for } k=2, \ldots, n
$$

and

$$
\begin{aligned}
\sum_{k=1}^{i}\left(a_{k}-a_{k+1}\right) k^{1-p / 2} & \leq a_{1}+\sum_{k=2}^{i} a_{k}\left(k^{1-p / 2}-(k-1)^{1-p / 2}\right) \\
& \leq a_{1}+\sum_{k=2}^{i} a_{k}\left(1-\frac{p}{2}\right)(k-1)^{-p / 2} \\
& \leq a_{1}+2\left(1-\frac{p}{2}\right) \sum_{k=2}^{i} a_{k} k^{-p / 2}
\end{aligned}
$$

By the hypothesis (1.2) we get

$$
\sum_{k=1}^{i}\left(a_{k}-a_{k+1}\right) k^{1-p / 2} \leq 2 C i^{1-p / 2} a_{i}
$$


Therefore we get, with a universal constant $D$,

$$
\begin{aligned}
& \left(\sum_{k=1}^{n-1}\left(a_{k}-a_{k+1}\right) k^{1-p / 2} \sum_{i=k}^{n} C_{i}\left|x_{i}^{*}\right|^{p}\right)^{1 / p} \\
& \leq C D\left(\sum_{i=1}^{n-1}\left|x_{i}^{*}\right|^{p} a_{i}+a_{n-1}\left|x_{n}^{*}\right|^{p}\right)^{1 / p} \cdot
\end{aligned}
$$

\section{2-CONCAVITY OF LORENTZ SPACES}

Proposition 4.1. Let $1 \leq p \leq 2$ and $1=a_{1} \geq a_{2} \geq \cdots \geq a_{n} \geq 0$. We have

$$
\begin{aligned}
& \operatorname{concave}_{2}(d(a, p)) \\
&=\sup \left\{\frac{\left(\sum_{i=1}^{n} a_{i}\left|b_{i}\right|^{p}\right)^{1 / p}}{\left(\sum_{i=1}^{n} a_{i}\left|c_{i}\right|^{p}\right)^{1 / p}} \mid\|b\|_{2}=\|c\|_{2},\right. \\
&\left.\sum_{i=1}^{k} b_{i}^{2} \geq \sum_{i=1}^{k} c_{i}^{2}, \begin{array}{l}
b_{1} \geq b_{2} \geq \cdots \geq b_{n} \geq 0, \\
c_{1} \geq c_{2} \geq \cdots \geq c_{n} \geq 0,
\end{array} k=1, \ldots, n\right\} .
\end{aligned}
$$

Compare also [13].

For the proof we require the following lemma which is essentially the same as Theorem 4.6 in [5].

Lemma 4.2. Let $x_{1} \geq x_{2} \geq \cdots \geq x_{n} \geq 0$ and $y_{1} \geq y_{2} \geq \cdots \geq y_{n} \geq 0$ such that

$$
\sum_{i=1}^{n} x_{i}=\sum_{i=1}^{n} y_{i}, \quad \sum_{i=1}^{k} x_{i} \geq \sum_{i=1}^{k} y_{i}, \quad k=1, \ldots, n .
$$

Then there are numbers $d_{r} \geq 0$ with $\sum_{r=1}^{N} d_{r}=1$ and permutations $\pi_{r}, r=$ $1, \ldots, N$, such that

$$
y_{k}=\sum_{r=1}^{N} d_{r} x_{\pi_{r}(k)}, \quad k=1, \ldots, n .
$$

Proof of Proposition 4.1. For simplicity we refer to the right-hand expression as $C_{2}$. We show first that the left-hand expression is smaller than the right-hand one. 


$$
\begin{aligned}
\left(\sum_{l=1}^{N}\left\|x_{l}\right\|^{2}\right)^{1 / 2} & =\left(\sum_{l=1}^{N}\left(\sum_{i=1}^{n}\left|x_{l}(i)^{*}\right|^{p} a_{i}\right)^{2 / p}\right)^{1 / 2} \\
& \leq\left(\sum_{i=1}^{n}\left|a_{i}\right|\left(\sum_{l=1}^{N}\left|x_{l}(i)^{*}\right|^{2}\right)^{p / 2}\right)^{1 / p} \\
& \leq C_{2}^{\sigma}\left(\sum_{i=1}^{n}\left|a_{i}\right|\left(\sum_{l=1}^{N}\left|x_{l}(\sigma(i))\right|^{2}\right)^{p / 2}\right)^{1 / p} .
\end{aligned}
$$

The last inequality holds because

$$
\sum_{i=1}^{k} \sum_{l=1}^{N}\left|x_{l}^{*}(i)\right|^{2}=\sum_{l=1}^{N} \sum_{i=1}^{k}\left|x_{l}^{*}(i)\right|^{2} \geq \sum_{l=1}^{N} \sum_{i=1}^{k}\left|x_{l}(\sigma(i))\right|^{2}
$$

is valid for all permutations $\sigma$. Therefore we get

$$
\left(\sum_{l=1}^{N}\left\|x_{l}\right\|^{2}\right)^{1 / 2} \leq C_{2}\left\|\left\{\left(\sum_{l=1}^{N}\left|x_{l}(i)\right|^{2}\right)^{1 / 2}\right\}_{i=1}^{n}\right\|
$$

Now we show the opposite inequality. Let $b, c \in \mathbf{R}^{n}$ with $\|b\|_{2}=\|c\|_{2}$ and

$$
\sum_{i=1}^{k} b_{i}^{2} \geq \sum_{i=1}^{k} c_{i}^{2}
$$

for all $k=1, \ldots, n$. By Lemma 4.2 we find numbers $d_{r}>0$ and $\sum d_{r}^{2}=1$ and permutations $\pi_{r}$ such that

$$
c_{k}^{2}=\sum_{r=1}^{N} d_{r}^{2} b_{\pi_{r}(k)}^{2}, \quad k=1, \ldots, n
$$

We have

$$
\begin{aligned}
\|b\| & =\left(\sum_{r=1}^{N} d_{r}^{2}\|b\|^{2}\right)^{1 / 2}=\left(\sum_{r=1}^{N}\left\|\left(d_{r} b_{\pi_{r}(k)}\right)_{k=1}^{n}\right\|^{2}\right)^{1 / 2} \\
& \leq \operatorname{concave}_{2}(d(a, p))\left\|\left\{\left(\sum_{r=1}^{N}\left|d_{r} b_{\pi_{r}(k)}\right|^{2}\right)^{1 / 2}\right\}_{k=1}^{n}\right\| \\
& =\text { concave }_{2}(d(a, p))\|c\| . \quad
\end{aligned}
$$

Lemma 4.3. Let $1 \leq p<2$ and $1=a_{1} \geq a_{2} \geq \cdots \geq a_{n} \geq 0$. Then we have $\left(\sum_{i=1}^{k}\left|a_{i}\right|^{2 /(2-p)}\right)^{(2-p) / 2} \leq\left(\operatorname{concave}_{2}(d(a, p))\right) k^{-p / 2} \sum_{i=1}^{k} a_{i}, \quad k=1, \ldots, n$.

Lemma 4.3 follows immediately from Proposition 4.1. 
Proposition 4.4. Let $a_{1} \geq a_{2} \geq \cdots \geq a_{n} \geq 0$ and $2 \leq s<\infty$ such that

$$
\left(\sum_{i=1}^{k}\left|a_{i}\right|^{s}\right)^{1 / s} \leq C k^{1 / s-1} \sum_{i=1}^{k} a_{i}, \quad k=1, \ldots, n \text {. }
$$

Then we have

$$
\sum_{i=1}^{k} a_{i} i^{1 / s-1} \leq D k^{1 / s-1} \sum_{i=1}^{k} a_{i}, \quad k=1, \ldots, n,
$$

where $D=D(C, s)$ does not depend on $n$.

Lemma 4.5. Let $a_{1} \geq a_{2} \geq \cdots \geq a_{n} \geq 0$ and $2 \leq s<\infty$ such that

$$
k^{1 / s-1} \sum_{i=1}^{k} a_{i} \leq C l^{1 / s-1} \sum_{i=1}^{l} a_{i}, \quad 1 \leq k \leq l \leq n .
$$

Then we have

$$
k^{1 / s-1} \sum_{i=1}^{k} a_{i} \leq 2(2 C)^{s /(s-1)} k^{1 / s} a_{k}, \quad 1 \leq k \leq \frac{n}{(2 C)^{s /(s-1)}} .
$$

Proof. Let $\alpha=\left[(2 C)^{s /(s-1)}\right]+1$.

$$
k^{1 / s-1} \sum_{i=1}^{k} a_{i} \leq C(\alpha k)^{(1-s) / s} \sum_{i=1}^{\alpha k} a_{i} \leq \frac{1}{2} k^{1 / s-1} \sum_{i=1}^{k} a_{i}+\frac{1}{2} k^{1 / s-1} \sum_{i=k+1}^{\alpha k} a_{i} .
$$

Therefore

$$
\frac{1}{2} k^{1 / s-1} \sum_{i=1}^{k} a_{i} \leq \frac{1}{2} k^{1 / s-1} \sum_{i=k+1}^{\alpha k} a_{i} \leq \frac{1}{2} k^{1 / s-1} \alpha k a_{k}=\frac{\alpha}{2} k^{1 / s} a_{k} .
$$

Lemma 4.6. Let $2 \leq s<\infty$ and $a_{1} \geq a_{2} \geq a_{3} \geq \cdots \geq a_{n} \geq 0$ such that

$$
\left(\sum_{i=1}^{k}\left|a_{i}\right|^{s}\right)^{1 / s} \leq C k^{1 / s} a_{k}, \quad k=1, \ldots, n .
$$

Then we have $a_{k} \leq \frac{1}{2} D^{1 / s} a_{D k}, 1 \leq k \leq n / D$, where $D \geq 2 \exp \left(2^{s} C^{2 s}\right)$ and $D \in \mathbf{N}$.

Proof. We have by hypothesis

$$
\sum_{i=1}^{k+j}\left|a_{i}\right|^{s} \leq C^{s}(k+j)\left|a_{k+j}\right|^{s} .
$$

This implies

$$
k\left|a_{k}\right|^{s} \leq C^{s}(k+j)\left|a_{k+j}\right|^{s}
$$

which implies

$$
C^{s} D k\left|a_{D k}\right|^{s} \geq \sum_{i=1}^{D k}\left|a_{i}\right|^{s} \geq \sum_{i=0}^{D k-k}\left|a_{k+i}\right|^{s} \geq C^{-s}\left|a_{k}\right|^{s} k \sum_{i=0}^{D k-k} \frac{1}{k+i} .
$$


Since

$$
\sum_{i=0}^{D k-k} \frac{1}{k+i} \geq \ln \left(\frac{D k}{k+1}\right) \geq \ln \frac{D}{2}
$$

we get $C^{2 s} D\left|a_{D k}\right|^{s} \geq\left|a_{k}\right|^{s} \ln \frac{D}{2}$. Since $D \geq 2 e^{2^{s} C^{2 s}}$ we get $\left(D / 2^{s}\right)\left|a_{D k}\right|^{s} \geq$ $\left|a_{k}\right|^{s}$.

Lemma 4.7. Let $2 \leq s<\infty, D \in \mathbf{N}$, and $a_{1} \geq a_{2} \geq \cdots \geq a_{n} \geq 0$ such that

$$
a_{k} \leq \frac{1}{2} D^{1 / s} a_{D k} \text { for } 1 \leq k \leq n / D \text {. }
$$

Then we have

$$
\sum_{i=1}^{k} a_{i} i^{1 / s-1} \leq D^{2} k^{1 / s} a_{k}, \quad 1 \leq k \leq \frac{n}{D} .
$$

Proof. We show by induction

$$
\sum_{i=1}^{D^{k}} a_{i} i^{1 / s-1} \leq D\left(D^{k}\right)^{1 / s} a_{D^{k}}, \quad 1 \leq D^{k} \leq n .
$$

We have

$$
\begin{aligned}
\sum_{i=1}^{D^{k+1}} a_{i} i^{1 / s-1} & =\sum_{i=1}^{D^{k}} a_{i} i^{1 / s-1}+\sum_{i=D^{k}+1}^{D^{k+1}} a_{i} i^{1 / s-1} \\
& \leq D\left(D^{k}\right)^{1 / s} a_{D^{k}}+a_{D^{k}}\left(D^{k}\right)^{1 / s-1}\left(D^{k+1}-D^{k}\right) \\
& =a_{D^{k}}\left(D^{k}\right)^{1 / s}(2 D-1) \leq a_{D^{k}}\left(D^{k}\right)^{1 / s} 2 D \leq a_{D^{k+1}}\left(D^{k+1}\right)^{1 / s} D .
\end{aligned}
$$

Proof of Proposition 4.4. By (4.2) we have in particular

$$
l^{1 / s-1} \sum_{i=1}^{l} a_{i} \leq C k^{1 / s-1} \sum_{i=1}^{k} a_{i}, \quad 1 \leq l \leq k \leq n .
$$

By Lemma 4.5 we get

$$
k^{1 / s-1} \sum_{i=1}^{k} a_{i} \leq 2(2 C)^{s /(s-1)} k^{1 / s} a_{k}, \quad 1 \leq k \leq \frac{n}{(2 C)^{s /(s-1)}} .
$$

This inequality together with (4.2) gives

$$
\left(\sum_{i=1}^{k}\left|a_{i}\right|^{s}\right)^{1 / s} \leq(2 C)^{s /(s-1)+1} k^{1 / s} a_{k}, \quad 1 \leq k \leq \frac{n}{(2 C)^{s /(s-1)}} .
$$

By Lemma 4.6 we get

$$
a_{k} \leq \frac{D^{1 / s}}{2} a_{D k}, \quad 1 \leq k \leq \frac{n}{D(2 C)^{s /(s-1)}},
$$

and $D$ is only depending on $C$. By Lemma 4.7 we obtain

$$
\sum_{i=1}^{k} a_{i} i^{1 / s-1} \leq D^{2} k^{1 / s} a_{k}, \quad 1 \leq k \leq \frac{n}{D(2 C)^{s /(s-1)}},
$$


which gives

$$
\sum_{i=1}^{k} a_{i} i^{1 / s-1} \leq D^{2} k^{1 / s-1} \sum_{i=1}^{k} a_{i}, \quad 1 \leq k \leq \frac{n}{D(2 C)^{s /(s-1)}} .
$$

For $k>n / D(2 C)^{s /(s-1)}$ we get the inequality because $a_{i}, i=1, \ldots, n$, is a decreasing sequence.

\section{REFERENCES}

1. J. Bretagnolle and D. Dacunha-Castelle, Application de l'étude de certaines formes linéaires aléatoires au plongement d'espaces de Banach dans les espaces $L^{p}$, Ann. Sci. Ecole Norm. Sup. (4) 2 (1969), 437-480.

2. J. Creekmore, Type and cotype in Lorentz $L_{p, q}$ spaces, Indag. Math. 43 (1981), 145-152.

3. D. Dacunha-Castelle, Variables aléatoires échangeables et espaces d'Orlicz, Séminaire MaureySchwartz 1974-75, exposés 10 et 11, Ecole Polytéchnique, Paris.

4. E. Dubinski, A. Pelczyński and H. P. Rosenthal, On Banach spaces $X$ for which $\pi_{2}\left(\mathscr{L}_{\infty}, X\right)=$ $B\left(\mathscr{L}_{\infty}, X\right)$, Studia Math. 44 (1972), 617-634.

5. G. H. Hardy, J. E. Littlewood and G. Polya, Inequalities, Cambridge Univ. Press, 1934.

6. W. B. Johnson, B. Maurey, G. Schechtman and L. Tzafriri, Symmetric structures in Banach spaces, Mem. Amer. Math. Soc. No. 217, 1979.

7. S. Kwapień and C. Schütt, Some combinatorial and probabilistic inequalities and their application to Banach space theory, Studia Math. 82 (1985), 91-106.

8. S_ Some combinatorial and probabilistic inequalities and their application to Banach space theory. II, preprint.

9. J. Lindenstrauss and A. Pelczyński, Absolutely summing operators in $L_{p}$-spaces and their applications, Studia Math. 29 (1968), 275-326.

10. J. Lindenstrauss and L. Tzafriri, Classical Banach spaces. I, II, Springer-Verlag, 1977 and 1979.

11. Y. Raynaud and C. Schütt, Some results on symmetric subspaces of $L^{1}$, Studia Math. 89 (1988), 27-35.

12. H. P. Rosenthal, On the subspaces of $L^{p} \quad(p>2)$ spanned by sequences of independent random variables, Israel J. Math. 8 (1970), 273-303.

13. S. Reisner, $A$ factorization theorem in Banach lattices and its application to Lorentz spaces, Ann. Inst. Fourier (Grenoble) 31 (1981), 239-255.

Department of Mathematics, Oklahoma State University, Stillwater, Oklahoma 74078 